\section{Re: The Emergence of Primary Care in Latin America: Reflections from the Field}

To the Editor: As a physician who was trained and is licensed in the United States and is also licensed in El Salvador and has practiced in mission medicine in rural El Salvador for 20 years, I enjoyed Dr. Ventres's ${ }^{1}$ perspective on primary care in Central America. I concur with that author's observation that El Salvador does a fine job of providing community-level primary health care. Community health care promoters are providing outstanding care related to universal vaccinations, prenatal health care, environmental interventions for outbreaks of dengue fever, and hospital referrals for emergencies. Being personally acquainted with some of these promoters, I also see the unfortunate side of the efforts to improve health care outcomes: these promoters, themselves generally from impoverished communities, are frequently threatened and sometimes penalized for negative outcomes beyond their control. These rural promoters perceive themselves as being virtually voiceless and powerless in a system where the lowest tier of workforce may be easily replaced by others eager for work.

However, for the many impoverished people who will die of acute and chronic disease if they are only vaccinated and given prenatal care, El Salvador is providing inadequate health care. Our clinic is barraged with patients who left the (free) public health care system because there is no metformin available for diabetes, who continue suffering from a wheezy cough despite having (inappropriately) received amoxicillin, and whose cancers are misdiagnosed and undertreated until treatment is no longer an option. Dr. Ventres teaches in San Salvador; I understand why he doesn't experience this daily aspect of the grave limitations of the health care system. At the time when El Salvador's care of acute/chronic diseases matches the wisdom and thoroughness of their prevention of diseases, we will have a model for the world.

Jana L. Nisly, MD

Amish Mennonite Aid, Santa Ana, El Salvador erclinicjn@gmail.com

\section{Reference}

1. Ventres WB. The emergence of primary care in Latin America: reflections from the field. J Am Board Fam Med 2013;26:183-6.

doi: 10.3122/jabfm.2013.05.130133

\section{Response: Re: The Emergence of Primary Care in Latin America: Reflections from the Field}

Dr. Nisly expresses frustrations about her daily work as a mission physician in El Salvador and notes several examples of how the health care sys- tem is failing there. She is absolutely correct in noting that the reality of health care in El Salvador is far from perfect. I suspect that pretty much the same could be said currently about any system in any place around the world.

That such frustrations and realities exist, however, should not blind us to the hope of building more rational systems of health care delivery. Efforts to develop community-based, locally accessible primary care services are worthy of support. Four years after the first peaceful democratic transfer of power in El Salvador's history, the current Ministry of Health is slowly making progress on its platform of reform. I applaud it for even trying.

More to the point for readers of the Fournal of the American Board of Family Medicine, every system of health care around the globe has weaknesses and strengths. Our greatest challenge as practitioners in the global community is to learn both from failures as well as successes-be they "theirs" or "ours"- and to acknowledge them honestly with measures of humility and respect. Above all else, that is the take home point from my article and this response.

William Ventres, MD, MA

Institute for Studies in History, Anthropology and Archeology, University of El Salvador San Salvador, El Salvador wventres@gmail.com doi: 10.3122/jabfm.2013.05.130193

\section{Re:Journal of the American Board of Family Medicine Issue on Communities of Solution}

To the Editor: We appreciated the Fournal of the American Board of Family Medicine issue (May/June 2013) dedicated to communities of solution (COSs) and believe that this topic enhances meaningful conversation around the role of health care institutions in improving individual and population health. Griswold et $\mathrm{al}^{1}$ highlighted key themes of COSs, such as crossing "jurisdictional boundaries" and community-based initiatives. The rest of the dedicated issue focused on specific examples of COSs related to these themes, including Ferrer et al's " ${ }^{2}$ advanced" multidisciplinary primary care and Garney et al's ${ }^{3}$ health resource centers. These examples are salient, 
but as clinicians we found that the articles failed to present a roadmap for describing how providers can break down barriers to intersectoral collaboration from within the health care setting to build COSs that address social determinants of health. We identify 3 major challenges to creating such a roadmap: (1) rigorous research and evaluation; (2) concrete strategies for dissemination and implementation of COSs; and (3) recommendations for sustainable funding.

There is a concerning lack of research about the most effective ways for health care institutions to address social determinants of health. Linkage programs such as those described by Ferrer et $\mathrm{al}^{2}$ and Garney et $\mathrm{al}^{3}$ have for the most part failed to be accompanied by rigorous evaluation to demonstrate health status or utilization outcomes. There is also a lack of evidence about how to build effective COSs across sectoral boundaries and a paucity of data about related population-level outcomes.

Furthermore, how can practitioners work to develop models that can be implemented, scaled, and disseminated? The health resource centers described by Garney et $\mathrm{al}^{3}$ link the delivery of health care and social services. This intervention reaches individual patients, geographic clinical catchment areas, and public health networks, but it offers insight into only one regional example rather than providing a tool kit that can be used by other regions. Garney et al conclude that solutions built and sustained within a COS are most effective. We would emphasize instead that interventions designed in external settings are not inauthentic but rather allow each community to base their efforts on an established framework. In fact, we believe that there should be networks in place to bridge learning and improve efficiency across different COSs, although there remain questions about what kinds of networks can be used and how recruitment, training, and data platforms can be shared across settings.

Finally, funding for innovative COS approaches that address social determinants of health in clinical settings is at best insufficient, although new demonstrations of payment reform are being explored in some state Medicaid programs. While there are studies suggesting that models for service linkage decrease ultimate health care costs, costs of implementation and concerns about ensuring subsequent reimbursement are major barriers.

These barriers are great, but so are the potential benefits of helping providers participate in COSs. Novel approaches to intersectoral collaboration are currently underway in programs around the country, some of which are described in the May/June issue of the fournal. Electronic health records and regional information networks provide additional opportunities to facilitate linkages across health and nonhealth settings. Important next steps include proving that these approaches make a difference in health outcomes for individuals and populations, articulating dissemination plans, and creating sustainable funding strategies. Such an approach will help to scale evidence-based COS models to regional and national forums.
Abby Burns, MDc, MSWc

School of Medicine and Center for Health and Community University of California, San Francisco Management and Planning, School of Social Welfare University of California, Berkeley abby.burns@ucsf.edu Laura M. Gottlieb, MD, MPH

Center for Health and Community Department of Family and Community Medicine University of California, San Francisco

\section{References}

1. Griswold K. S, Lesko SE, Westfall JM. Communities of solution: partnerships for population health. J Am Board Fam Med 2013;26:232-8.

2. Ferrer RL, Gonzalez Schlenker C, Lozano Romero R, et al. Advanced primary care in San Antonio: Linking Practice and Community Strategies to Improve Health. J Am Board Fam Med 2013;26:288-98.

3. Garney WR, Drake K, Wendel ML, McLeroy K, Clary HR, Ryder B. Increasing access to care for Brazos Valley, Texas: a rural community of solution. J Am Board Fam Med 2013;26:246-53.

doi: 10.3122/jabfm.2013.05.130171

\section{Response: Re:Journal of the American Board of Family Medicine Issue on Communities of Solution}

We appreciate the thoughtful comments of Burns and Gottlieb ${ }^{1}$ and agree with many of their points. Throughout our work examining the Folsom Report, ${ }^{2}$ we have noted many of the same pitfalls regarding current fledgling communities of solution (COSs): the lack of rigorous research, outcomes data, and nationwide or regional networks. Burns and Gottlieb's cogent suggestions to further the rigor of COSs and assess outcomes are critical next steps.

Health care providers are crucial members in a COS. While some barriers to provider inclusion do exist, providers often choose not to participate because of other pressing work or lack of payment for community-focused work. Alternatively, providers do not necessarily have to lead a COS but can join instead as partners. Groups may more willingly add providers to their invite lists if the providers are merely one of the stakeholders.

The concept of any particular local COS is not always scalable; it may not provide evidence for the same solution in another COS. However, there is a need for a lattice that can connect COSs for ideas, support, funding, research.

Health insurers and hospitals also play an important role in concordance with their mandates for community benefits. Funding agencies and foundations could consider supporting a national research network to inform the coalition of public health and primary care across 